\title{
Real-Time Issues in Mobile Wireless Networks
}

\author{
Giorgio Buttazzo \\ Scuola Superiore Sant'Anna, Pisa
}

The use of cooperating mobile robots is requested in an increasing number of application domains, including civil protection, surveillance, environmental monitoring, under-water exploration, and space missions. In most of these applications, the robot units are required to acquire sensory information, localize themselves in the environment, plan trajectories, avoid obstacles, and cooperate with the other robots to reach a common goal.

To achieve such objectives, the development of a team of cooperating robots poses several interesting problems from a research point of view, such as the realtime execution of acquisition and control processes, the efficient management of computational resources, the software control of energy consumption, the realtime communication protocols on wireless networks, and the development of distributed agreement algorithms for reaching a consensus in collective decisions. Moreover, small mobile robots are often controlled by micro-controllers having low computational power and limited resources, hence satisfying timing constraints requires the use of efficient operating systems and algorithms that can guarantee a predictable behavior both in normal and overload conditions.

This talk will present some of the most challenging problems to be solved in order to support the development of mobile wireless networks of cooperating robots. 